\title{
Le management des interventions policières en contexte congolais. Réflexion sur l'administration du commandement
}

\author{
Par LUPITSHI WA NUMBI Norbert*, KALALA ILUNGA Matthiesen** et \\ MUMBA KAKUDJI Martial ${ }^{* * *}$
}

\section{A. Introduction : annonce du thème traité et son questionnement}

Le titre du présent article suscite curiosité et intérêt mais se révèle aussi quelque peu osé. A sa première lecture, on serait probablement tenté d'attendre du développement réflexif de l'auteur la proposition d'une recette managériale toute faite de conduite de l'action ou de gestion des structures sécuritaires. Il nous semble donc bien propice de commencer par étaler la double ambition de cette petite dissertation : d'une part, elle tente de tirer, à l'attention des acteurs sécuritaires congolais en général et policiers en particulier, une sonnette sur la délicatesse de la question managériale dans leurs structures professionnelles et d'autre part, il cherche à susciter un débat scientifique dans le milieu des chercheurs sur une autre "possible » manière de penser l'action sécuritaire en contexte congolais, un contexte extraordinairement singularisé, comme nous le montrons ci-dessous, par des pesanteurs de plusieurs ordres et de tout acabit. La question managériale soumise à l'examen, se révélant trop complexe, le contour de l'analyse faite mérite également circonscription : il s'articule autour de la conduite du commandement, entendu ici comme " principal ressort » des politiques et des actions menées au sein des unités et/ou des structures sécuritaires. Il importe de reconnaitre que le commandement des unités sécuritaires, au centre de la présente analyse, a de sérieuses conséquences sur les droits et les devoirs des acteurs impliqués, selon la manière dont il est administré et/ou exécuté.

Dans la vie courante, il est des observateurs pour qui les dirigeants des unités sécuritaires ne peuvent aucunement se prévaloir le rôle de planificateurs à cause, estiment-ils, de la centralisation à outrance de ces unités et de leur forte dépendance à l'autorité politique. Et pourtant, note TSHINYAMA KADIMA (2009) ${ }^{1}$, « les dirigeants de la police sont aussi jugés selon leurs capacités managériales, c'est-à-dire leur capacité à organiser et à faire fonctionner cette structure ». Comme d'aucuns peuvent le constater, la question managé-

* Professeur à l'Université de Lubumbashi, Docteur en criminologie.

** Professeur Ordinaire à l'Université de Lubumbashi.

*** Chef de Travaux à la Faculté de Droit de l'Université de Lubumbashi, DEA en Droit Public et Avocat au Barreau de Lubumbashi.

1 TSHINYAMA, KADIMA (2009), L'observation ethnographique d'un commissariat à Lubumbashi. Une compréhension des pratiques policières en contexte congolais, Thèse de doctorat en criminologie, Université de Lubumbashi (RDC). 
riale devient alors importante et pertinente à décortiquer scientifiquement dans le fonctionnement des structures sécuritaires en général et particulièrement dans celles de la RDC, notre pays.

Aux dires de certains officiers de police interrogés ${ }^{2}$, le commandement, bien que parfois orienté et limité par d'autres instances hiérarchiques, repose officiellement sur trois éléments suivants : la définition des buts au regard de la mission attribuée à l'unité; la coordination des activités de l'unité qui implique un arbitrage entre forces centripètes et centrifuges et le contrôle du fonctionnement quotidien de l'unité. Nous trouvons que pour être efficaces, cette coordination, cet arbitrage et ce contrôle appellent un management, c'est-àdire un recours à certaines techniques de direction et de gestion car, comme l'indique Paolo NAPOLI $(2003)^{3}$, « la notion de sécurité a [de nos jours] perdu sa connotation exclusivement psychologique et a acquis une dimension objective, mesurable » et donc planifiable . $^{4}$

Sur le plan méthodologique, la question à la base de cette étude se formule de la manière suivante : comment penser l'administration du commandement dans les structures sécuritaires en contexte congolais?

La question soulevée trouve, d'une part, sa quintessence dans la thèse largement répandue en sociologie policière selon laquelle « les pratiques policières [ou sécuritaires] reflètent les valeurs et les normes qui prévalent dans une société » et d'autre part, dans la montée des « désordres urbains» ${ }^{5}$ et celle des statistiques criminelles et pénitentiaires qui rendent nécessaire le management de l'action sécuritaire.

Sur le plan théorique, la réflexion développée relève - bien entendu - de la sociologie policière, une sociologie dont les travaux s'inscrivent généralement dans deux grandes orientations : celle qui procède à l'analyse du cadre institutionnel, organisationnel ou professionnel et celle qui privilégie les pratiques des acteurs, comme cadre d'observation et d'analyse [l'approche par les acteurs] $]^{6}$. La presque majorité des recherches menées à l'école de criminologie de l'université de Lubumbashi s'inscrivent dans la deuxième orientation. Rares sont donc les recherches qui s'insèrent dans l'orientation managériale. Il se révèle alors tout à fait justifié de tenter de captiver la curiosité des étudiants et des chercheurs par rapport à cette orientation managériale visiblement peu exploitée dans la pratique criminologique en RDC.

Hormis l'introduction (entamée), l'essentiel de notre propos s'articule autour de quatre petits points suivants : 1) le contexte d'émergence de l'objet traité et la méthodologie utilisée; 2) la logique fonctionnelle des corps ou des structures sécuritaires; 3) les tensions dans

2 Les officiers de la police des mines et de la circulation routière (de Lubumbashi), deux des branches spécialisées de la police nationale congolaise.

3 Paolo NAPOLI (2003), Naissance de la police moderne, Paris, La Découverte.

4 Terme ajouté par nous.

5 Par "désordres urbains", nous entendons toutes les situations-problèmes dérangeant l'ordre et la sécurité publique » dans nos villes.

6 TSHINYAMA, KADIMA. (2009), op.cit. 
la conduite de l'action sécuritaire et 4) l'esquisse de ce qui correspondrait au « management de l'action sécuritaire en milieu congolais (notre suggestion) ».

\section{B. Le contexte d'émergence du thème traité et la méthodologie de travail}

Ce texte est, au fait, la systématisation de plusieurs conférences et interventions que nous organisons avec les étudiants de l'école de criminologie de l'Université de Lubumbashi ainsi qu'avec les médias autour de la thématique de « réforme sécuritaire, c'est à dire policière et militaire, en République démocratique du Congo ». Ces activités de réforme de la police et de l'armée en RDC, constituent donc le champ-contexte dans lequel s'insère l'objet ou la question soulevée, en l'occurrence l'administration ou la gestion du commandement dans les structures sécuritaires de la $R D C$. La pertinence et le haut niveau des débats qu'ont occasionné nos interventions constituent le leitmotiv direct qui nous a poussé à envisager l'enrichissement et l'approfondissement de notre réflexion sur cette problématique. Les questions soulevées lors de différents échanges entretenus et les remarques nous administrées déterminent pour une bonne part la structuration et le contenu du présent texte.

S'il faut placer un mot sur le rapport du chercheur à la question traitée, on notera que ce sujet prend ses ramifications lointaines dans notre recherche de DEA portant sur les «pratiques non réglementaires observées dans les transports en communs à Lubumbashi $»^{8}$. Dans certains de ses aspects, cette étude a eu l'avantage de dévoiler différentes contraintes qui pèsent sur les policiers congolais commis à la régulation de la circulation routière dans l'accomplissement de leurs tâches. Le poids de ces contraintes a fait que la plupart des commandements institutionnels reçus avaient du mal à être exécuté à la lettre par les policiers; elles se sont, dans une large mesure, révélées inopérantes. Cet état de la situation [la difficulté d'application des ordres institutionnels sur le terrain] est resté gravé dans notre mémoire et a resurgi lorsque l'offre de communication nous a été adressée à l'occasion des journées scientifiques sus-évoquées.

Le processus de systématisation de notre réflexion, pour lui donner les caractères d'un texte scientifique, nous a demandé d'une part, d'éplucher la littérature tant nationale qu'étrangère sur le sujet abordé et d'autre part de procéder à des entretiens actualisés avec les officiers de commandement des unités de police et de l'armée. Probité intellectuelle oblige, nous devons avouer que l'examen de la littérature antérieure a été sélectif mais bien ciblé, au local et à l'international. Quelques auteurs canadiens et européens ont été exploités. Puisqu'il s'agit d'un sujet qui traite de la réalité congolaise, la recherche criminologique de TSHINYAMA KADIMA (2009) ainsi que les mémoires des étudiants de l'école de criminologie de l'UNILU ont largement été mis à contribution. En outre, il sied égale-

7 Luc ALBARELLO (2003), Apprendre à chercher. L'acteur social et la recherche scientifique, Bruxelles, De Boeck, $2^{\mathrm{e}}$ édition.

8 LUPITSHI WA NUMBI (2006), Les pratiques non règlementaires dans le réseau automobile des transports en commun de Lubumbashi, Mémoire de DEA en criminologie, Université de Lubumbashi. 
ment de souligner que notre effort réflexif pèse beaucoup dans la mise en musique des données livresques et des données d'entretien.

Au chapitre des entretiens proprement dits, la tâche nous a paru on ne peut plus aisée, car ceux-ci ont été organisés, nous le rappelons, avec les officiers de la police et de l'armée, étudiants et anciens étudiants de l'école de criminologie de l'UNILU en licence et au DEA $^{9}$. Les syntagmes de commandement commentés dans ce texte nous ont été livrés et expliqués par eux. Nous recevions ces officiers-étudiants dans notre bureau de travail soit avant, soit après les cours qu'ils venaient suivre mais c'était, chaque fois, sur rendez-vous préalablement négocié et fixé. Nous ne pouvons pas non plus manquer de relever le fait que l'« heureux hasard » nous a aussi offert quelques rencontres fortuites avec les enquêtés que nous avions vite capitalisées et exploitées. La majorité des officiers interrogés appartiennent à la police des mines et à la police de circulation routière. Au plan fonctionnel de ces deux branches de la police nationale congolaise, il est également important de souligner le fait que malgré les tâches déjà spécifiées pour chacune, celles-ci restent néanmoins bien ancrées dans la « haute » sécurité de l'Etat.

Quant à leur contenu, ces entretiens ont porté notamment sur leur formation policière et/ou militaire ${ }^{10}$, sur l'administration du commandement et sur l'exécution [problématique] de leurs ordres par les éléments sous leur commandement. Nous ne manquerons pas non plus d'avouer que certains de ces entretiens n'ont pas été bien formalisés au moyen un guide d'entretien savamment élaboré, ils se sont réalisés sous forme d'échanges ouverts et libres mais portant sur des aspects et des détails concordants à l'objet sous examen. Au sujet de la formation reçue par ces officiers, deux tendances ont été relevées : les uns [la tendance minoritaire] sont les produits de l'ancienne garde civile créée vers les années 80 par feu Maréchal Mobutu et les autres [la tendance majoritaire] proviennent des éléments de 1'AFDL ${ }^{11}$ de Kabila-père des années 19996-1997. Passé sous un filtre critique, ce petit détail donnerait une idée sur les tractations qu'a connues et que connait peut-être encore la RDC dans le brassage de différentes composantes de son armée. Par rapport à la gestion du commandement, presque tous les enquêtés ont reconnu que toute intervention policière ou sécuritaire se fait sur ordre de la hiérarchie qui précise aussi les objectifs à atteindre dans chaque situation ${ }^{12}$. Et en ce qui concerne l'exécution des ordres reçus par les policiers, les propos des enquêtés se recoupaient beaucoup plus sur les aspects relatifs à la difficulté et/ou à la délicatesse de leur exécution suite justement aux contraintes analysées plus bas.

9 Pour question d'éthique, nous avons tu les noms de nos enquêtés.

10 Il faut aussi signaler qu'avec l'avènement du régime de Kabila père, une certaine porosité a été observée entre la police et l'armée et cela, dans les deux sens.

11 Alliance des forces démocratiques pour la libération du Congo.

12 Allusion faite ici aux propos du commandant urbain de la police ou brigade d'intervention rapide. 


\section{La logique fonctionnelle des corps sécuritaires}

Il est, de façon générale, conféré aux structures sécuritaires le statut de « corps assimilés à l'armée ». Dans plusieurs sociétés, indique LUNSUA KINZENZE. (2009)13, les organisations policières fonctionnent sur le mode des unités de l'armée. Cela conduit à des modes d'intervention privilégiant une distance vis-à-vis du public et le langage policier en est témoin. Lorsqu'on intervient sur terrain, on parle ainsi de « zones hostiles ${ }^{14}$ », de « manifestations hostiles », d'《 ennemis de la paix », etc. Cet état des choses entraine des modes d'intervention de type militaire qui ne privilégient pas le contact et la collaboration avec la population, pourtant indispensable et voire incontournable.

En tant que corps assimilés à l'armée et vu aussi leurs solides accointances avec le pouvoir politique en place, les corps sécuritaires [dont la police] connaissent, au niveau des rapports interprofessionnels et hiérarchiques un commandement plus ou moins carré, rigide qui dénote justement de cette logique militaire, et on arguera que cette rigidité serait liée à la discipline de fer censée caractériser ces genres d'unités et cela se lit clairement dans la nomenclature sanctionnant les grades. C'est ainsi, par exemple, qu'au niveau des Officiers, un Commissaire Divisionnaire en Chef préfère être appelé «Lieutenant Général », un Commissionnaire Divisionnaire préfère « Général Major », Un Commissaire Divisionnaire Adjoint « Général de brigade » et un Commissaire Supérieur Principal trouve mieux de se faire appeler «Colonel », etc. TSHINYAMA KADIMA $(2009,330)^{15}$ indique que la police congolaise, bien que disposant d'une nomenclature des grades spécifiques, valorise plutôt la nomenclature militaire et ceci, dans le souci de renforcer les rapports d'autorité entre officiers et subalternes. Dans sa concrétude, ce type de commandement carré se traduit, dans le contexte congolais, par divers syntagmes autoritaires [généralement véhiculés en Lingala, langue usuelle dans l'armée] dont notre sélection ci-après :

1) «Règlement eza monoko ya mokonzi », c'est-à-dire : " la parole du chef vaut le règlement $»$;

2) «Discipline eza kotosa, kozongisa monoko te, eza komemya bakonzi », qui veut dire : « La discipline, c'est obéir, ne pas répliquer aux ordres du chef, c'est se soumettre »;

3) «Oza raisonneur! » [Tu raisonnes trop!], « Bomba mayele nayo na poche » [Garde ton intelligence en poche], "Policier azalaka matoyi na miso monene, kasi azalaka na monoko muke » [le policier a de grandes oreilles pour écouter et de gros yeux pour observer mais une petite bouche pour « répliquer peu »] $(\ldots)^{16}$.

13 LUNSUA KINZENZE (2009), La restructuration des relations dans une unité de police. Un mode de gestion du commandement, mémoire de licence en criminologie, Ecole de criminologie, Université de Lubumbashi (RDC).

14 Qui veut dire ennemi.

15 TSHINYAMA KADIMA (2009), op.cit.

16 Les travaux des chercheurs et étudiants de l'Ecole de criminologie de l'UNILU livrent plusieurs autres syntagmes analogues sur les rapports hiérarchiques dans les corps sécuritaires fonctionnant en RDC. 
Et la réalité de terrain montre encore mieux qu'à chaque ordre reçu, le policier ou l'agent sécuritaire subalterne réplique : «A vos ordres! Mokonzi (Chef)! Mon Général! Mon Colonel! Mon Capitaine! et cela, selon le grade de l'officier émetteur dudit ordre.

Loin de nous la prétention d'une quelconque méconnaissance de l'utilité voire la nécessité d'un commandement bien fort et d'une discipline exemplaire dans les structures sécuritaires, la lecture criminologique de cette réalité montre que ce genre de commandement tend à une sorte de « robotisation » de l'action policière. Le policier ou l'agent sécuritaire est présenté à l'image d'un acteur automate qui répercuterait sans vergogne, automatiquement et fidèlement les préceptes, les ordres ou les consignes du « chef». Nous sommes ici en face d'une vieille conception fondée sur le présupposé que « c'est la loi ou la consigne hiérarchique qui règle l'action sécuritaire ou policière ». Cette conception tendant à réduire le fonctionnement des corps sécuritaires à leur seul aspect institutionnel, a jadis, amené plusieurs sociétés, en particulier les sociétés anglo-saxonnes, à prendre la police pour un simple « instrument spécialisé d'application de la loi ». De nos jours, il est fort probable de trouver des sociétés encore bien ancrées dans cette conception et cette logique fonctionnelle.

Dominique MONJARDET $(1996,34)^{17}$ trouve qu' « il n’y a pas de police « presse-bouton » que son chef [Directeur, Commissaire, Inspecteur, Ministre, Maire de la ville, etc.] actionnerait à loisir dans une transparence entière et avec une parfaite adéquation entre intention et résultat ». C'est ainsi que dans une appréhension ouverte, Jean-Paul BRODEUR et Dominique MONJARDET (2003) ${ }^{18}$ mettent en exergue trois facettes de la police, en tant que corps sécuritaire : police comme organisation, police comme institution et police comme profession. Ces facettes font que la police prend un visage extrêmement complexe et ses actions répondent, de ce fait, à des logiques plurielles.

Pour reprendre la réflexion de ces auteurs, en tant qu'organisation, la police est appréhendée dans sa facette formelle : une structure, un organigramme, des ressources humaines et matérielles et leur agencement selon les règles explicites qui prescrivent la façon dont cette organisation doit opérer. Comme institution, la police est un organe qui veille au respect de la loi et donc un instrument de mise en œuvre de la force coercitive et comme profession, la police est un système de normes et des valeurs sous-tendant les comportements des policiers. Ces valeurs et normes, représentant ce que certains auteurs qualifient de culture professionnelle, reposent, entre autres, sur les principes de solidarité, sur l'appartenance à une même structure et sur le partage d'une identité commune.

La vision ouverte des corps sécuritaires telle que soutenue par Jean-Paul BRODEUR et Dominique MONJARDET débouche sur la mise en évidence d'une gamme variée des tensions ou des contraintes qui s'exercent sur les agents sécuritaires dans la conduite de leurs

17 Dominique MONJARDET (1996), Ce que fait la police. Sociologie de la force publique, Paris, La Découverte.

18 Jean-Paul BRODEUR, et Dominique MONJARDET (2003), " connaitre la police " dans BRODEUR, J.P. et MONJARDET, D. (dir), Connaitre la police. Grands textes de la recherche anglosaxonne, Paris, IHESI, pp. 9-21. 
actions. Ces pressions qui méritent un dévoilement, constituent à notre entendement, des facteurs que les instances de commandement ne peuvent pas se permettre d'ignorer à l'initiation et à l'administration du commandement.

\section{Des tensions dans la conduite de l'action sécuritaire en contexte congolais}

Le contenu de ce point tente de montrer que l'agir du policier ou de l'agent sécuritaire ne relève ni du seul réflexe institutionnel ni de son " seul libre arbitrage »; il est à situer au carrefour ou à l'intersection de plusieurs pressions à la fois internes et externes. Lenoir LAROCHE repris par Paolo NAPOLI $(2003,244)^{19}$ trouve que la considération de la situation contingente reste le critère fondamental de la conception de l'action policière. Or, les contingences situationnelles font que les mêmes règles, les mêmes principes, les mêmes modèles peuvent connaître une interprétation et une application différenciées. C'est alors que l'action sécuritaire ou policière devient délicate à mener et appelle, de ce fait, un management au commandement et à l'exécution des ordres intimés.

Inter-reliées et relevant, comme déjà affirmé, de plusieurs ordres, ces contraintes peuvent se résumer comme suit: pluralité d'ordres normatifs, pluralité de registres, pluralité de normes référentielles, pluralité de rationalités et des logiques, etc. Dans le réseau automobile des transports en commun de Lubumbashi (en RDC) ${ }^{20}$, par exemple, le tableau « rigide » des contraventions routières à appliquer institutionnellement par les policiers de régulation routière fait concurrence farouche avec diverses pratiques et normativités de terrain au point que le réglementaire et l'illicite s'interpénètrent et co-fonctionnent dans les rouages dudit réseau. Tellement imbriqués ces deux types de normativités semblent fonctionnellement bien se soutenir l'un et l'autre. En pareil contexte, estiment bien des auteurs dont LUNSUA KINZENZE $(2009,99)^{21}$, il est difficile d'établir un distinguo entre le formel et l'informel; ce " clivage » doit être dépassé pour laisser place à une structure particulière de régulation interne qui tient compte de l'inventivité de tous les acteurs, dirigeants [commandants] y compris.

Pour sa part, TSHINYAMA KADIMA $(2011,164)^{22}$ constate que l'agent sécuritaire congolais chargé officiellement de la répression est plutôt perçu par sa société comme un agent communautaire chargé, entre autres, de la résolution des conflictualités sociales, il s'adonne dans ce cas-ci au "social service ». Le même agent, en tant qu'acteur social, nourrit des projets et des intérêts personnels qu'il tient à concrétiser nonobstant les pressions à gérer, c'est le self-service. Dans ce même ordre d'idées, Dominique MONJARDET

19 Paolo NAPOLI (2003), op.cit.

20 LUPITSHI WA NUMBI (2006), op.cit.

21 LUNSUA KINZENZE (2009), op.cit.

22 TSHINYAMA KADIMA (2011), " comprendre les pratiques policières en contexte congolais. Une étude ethnographique réalisée dans un commissariat de Lubumbashi », in Déviance et société, La charte, $\mathrm{pp} . .$. 
$(1996,145)^{23}$ souligne qu'outre les prescriptions hiérarchiques et le mandat social, les policiers sont également orientés par des intérêts professionnels propres. Par conséquent, le fonctionnement policier devient incompatible [inefficace] si on ne prend pas en compte les dimensions pertinentes de cette profession, les propriétés et les utilités " particulières » mais collectives de ceux à qui cette force publique est confiée.

En plus, sociologiquement parlant, le jeu social ne trouvant véritablement de sens que dans la connexité des faits et l'interrelation des sujets, ceci fait que beaucoup d'acteurs sociaux dont les agents sécuritaires agissent souvent en réseau pour se soutenir mutuellement. C'est ici que la ressource dite « savoir vivre ensemble » développée par Raoul KIENGE $\mathrm{KIENGE}^{24} \mathrm{~s}$ 'impose à tous. L'action sécuritaire à mener ou l'ordre hiérarchique reçu devient raisonné(e), car on doit tenir compte d'autres inter actants, on doit apprendre à bien faire avec les autres, c'est ce que TSHINYAMA KADIMA (2011) nomme le co-service. Sous ce registre dit co-service, les pratiques et les règles du jeu se rapportent au réseautage social et ce, dans une logique plurielle : de sociabilité, de réciprocité, d'assistance, de sécurisation professionnelle, etc. De ce fait, ce réseautage, à caractère mutualiste, devient une sorte de ressource parallèle aux voies officielles permettant en même temps aux acteurs d'atteindre certains intérêts et au système de fonctionner.

A ce premier paquet de contraintes, s'ajoutent les pressions d'ordre matériel et logistique telles qu'elles se vivent dans la presque totalité des services publics congolais. A ce tableau de contraintes, on retient notamment la précarité du travail, la modicité des salaires payés, la rareté et/ou la vétusté de l'équipement et du matériel de travail, l'insuffisance de formation et le manque de motivation des agents, etc. ${ }^{25}$. Une autre pression vient de la communauté internationale agissant via ses différentes composantes [Human right, MONUS$\mathrm{CO}$, etc.] et de la société civile qui veillent - tant bien que mal - à la défense et au respect des droits humains. En effet, ces agences internationales épient les agents sécuritaires dans leurs interactions avec la population. Une intervention, une application aveugle d'un ordre reçu pourrait coûter cher et même très cher à l'agent exécutant qui se rendrait coupable de violation prouvée des droits humains sous l'œil et/ou la caméra de l'un des organismes des Nations-Unies. Une dernière contrainte tout aussi remarquable se rapporte à l'immixtion et à l'imprévisibilité de l'autorité politique congolaise dont les choix décisionnels se révèlent souvent très fluctuants. Les animateurs-responsables des structures publiques congolaises, en général, et sécuritaires, en particulier, fonctionnent dans une sorte de «qui-vive » permanent car, de façon inopinée, une décision de destitution, de remplacement ou de permutation reste une éventualité.

23 Dominique MONJARDET (1996), op.cit.

24 Raoul KIENGE KIENGE (2005), Le contrôle policier de la délinquance des jeunes à Kinshasa. Une approche ethnographique en criminologie, thèse de doctorat, Université Catholique de Louvain.

25 Pour plus d'information, lire Tréfon THEODORE (2007), Parcours administratifs dans un Etat en faillite. Récits populaires de Lubumbashi (RDC), Paris, L'Harmattan. 
Pour Jean-Noël TREMBLAY $(1997,232)^{26}$, le métier de policier dépasse aujourd'hui le domaine strictement légal; il doit absolument intégrer la vie sociale et culturelle de la communauté afin de pouvoir agir adéquatement. Presque dans ce même angle réflexif, Robert CASTEL $(1990,300)^{27}$ estime que la rationalité juridique doit toujours être un travail d'ajustement de la règle aux circonstances.

Coincé dans cette trame de tensions, l'agir de l'agent sécuritaire congolais relève d'un perpétuel et indéfini « bricolage » teinté à la fois d'ingéniosité et d'imprédictibilité. Dans sa prestation, il procède à des adaptations et à des ajustements innovants [au sens mertonien]; il est constamment partagé entre l'Etat, son employeur [application stricte des règles et des ordres reçus], lui-même [ses projets et ses intérêts], les partenaires de service [au titre du bien vivre ensemble] et la société.

Pour corroborer ce qui précède, Jean-Noël TREMBLAY (1997) se résume en disant qu'au cœur du management policier [ou sécuritaire] se trouve le travail du dirigeant [commandant]. Le commandement policier, renchérit-il, peut donc faire l'objet de management, un management conçu en fonction de la complexité et de la diversité des tensions qui s'exercent sur les agents sécuritaires.

La pluralité et l'ampleur des pesanteurs évoquées donnent déjà matière à réflexion sur ce que serait la manière de mieux penser le management du commandement sécuritaire en RDC. Voilà qui nous conduit à l'abord du point de chute du présent article.

\section{E. Penser le management du commandement dans les structures sécuritaires en contexte congolais}

Si nous souscrivons à la pensée selon laquelle « la police est ce que la société veut qu'elle soit », cela pousserait à la recherche et à l'identification de quoi ou de qui [ou plutôt de ceux] se cacheraient derrière ce vocable flou et englobant de « société ».

Faisant du management de commandement sécuritaire l'épicentre de notre analyse, nous voulons insister sur le fait que, c'est ce commandement qui, en principe, constitue le principal vecteur de l'action ou de l'activité sécuritaire. Le vieux dicton sécuritaire mérite, en cette occurrence exhumation : " il n'y a pas de mauvaises troupes, il n'y a que de mauvais chefs » et ceci, tout simplement parce que, c'est le chef qui commande, initie et planifie les actions à mener sur le terrain. Analysant le mode de gestion du commandement dans l'unité de police d'investigation criminelle de Lubumbashi (RDC), LUNSUA KINZENZE $(2009,99)^{28}$ reconnait que le commandant d'une unité sécuritaire a des buts à atteindre. Sa carrière d'officier dépend largement des décisions qu'il prend suscitant certes diverses réac-

26 Jean-Noël TREMBLAY (1997), Le métier de policier et le management, Canada, Presses de l'Université de Laval.

27 Robert CASTEL (1990), «L'application de la loi : l'ordre des interactions et l'ordre des déterminations », in DIGNEFFE, F. (dir), Acteur social et délinquance. Une grille de lecture du système de justice pénale, Bruxelles/Liège, Mardaga, Coll. Psychologie et Sciences humaines, pp.295 - 303.

28 LUNSUA KINZENZE (2009), op.cit. 
tions. Celui-ci, continue le même auteur, doit savoir que l'unité qu'il commande n'est pas seulement un système social mais un organisme dont l'efficacité est liée à ses décisions. En plus, son unité [sa branche de commandement] n'est pas isolée de la structure-mère [la police nationale] ou de la société. Le commandant consacre donc son activité au fonctionnement technique et à l'exercice des attributs de pouvoir structurel en s'adaptant à certaines conditions de la vie sociale du milieu dans lequel ladite unité évolue. Il nous semble alors assez logique de rejoindre une fois encore Jean-Noël TREMBLAY ${ }^{29}$ pour qui, au cœur $d u$ management des structures sécuritaires, il importe " d'insister sur le travail de commandement».

Or, il a été précédemment indiqué que, malgré les prescriptions hiérarchiques - théoriquement définies - l'action sécuritaire est largement dépendante des pressions de divers ordres au point que Dominique MONJARDET $(1996,34)^{30}$ en est arrivé à la récusation d'une police « presse-bouton » de laquelle on attendrait une parfaite adéquation entre intention [commandement reçu] et résultat. C'est ainsi que Paolo NAPOLI $(2003,245)$ estime qu'en matière de commandement, la police moderne [en tant que corps sécuritaire] requiert moins de prescriptions et plus de savoir-faire; elle doit plus viser au compromis qu'à l'injonction. Dans ce même ordre de raisonnement, TSHINYAMA KADIMA (2011) trouve que le travail au quotidien de l'agent sécuritaire congolais se révèle (être) un véritable « aménagement » entre multiples contraintes d'ordre organisationnel, professionnel, institutionnel, politique, social et individuel.

Selon notre entendement, nous lisons plutôt dans cet « aménagement » la possibilité de rabibocher diverses pesanteurs souvent présentées comme « agissant toujours en sens opposé ». Contrairement à Dominique MONJARDET (1996) qui constate l'existence de tension [une sorte d'opposition permanente] $]^{31}$ entre les trois facettes de la police déjà évoquées [organisation, institution et profession], tension au cours de laquelle « la dimension institutionnelle serait toujours déformée dans son application » au point que « les buts formels seraient toujours détournés au profit des utilités policières particulières $[\ldots] »$, nous trouvons, par contre en cela, une imbrication fonctionnelle sui generis des pratiques et des logiques relevant de chacune de ces trois dimensions [facettes]. Notre lecture soutient alors que les structures sécuritaires, en l'occurrence la police, peuvent fonctionner dans un cycle particulier tantôt comme organisation, tantôt comme institution mais aussi comme profession sans qu'il y ait toujours tension. Parfois, dans un particulier dynamisme situationnel, le tout peut bien s'engrener, s'interpénétrer, comme le soutient aussi TSHINYAMA KADIMA (2011, 167) à l'aide d'un schéma interprétatif à travers lequel les pratiques organisationnelles, ins-

29 Jean-Noël TREMBLAY (1997), op cit.

30 Dominique MONJARDET (1996), Ce que fait la police. Sociologie de la force publique, Paris, La Découverte.

31 Notre interprétation. 
titutionnelles et professionnelles des agents sécuritaires se développent comme des « allant de soi ${ }^{32}$, donc comme des réalités qui ne se contredisent pas forcément.

$\mathrm{Vu}$ le constat établi ainsi que les éclairages apportés ci-dessus, si nous revenons au questionnement central soulevé, une investigation osée consisterait alors à tenter de dévoiler « le visage d'un possible management du commandement dans les structures sécuritaires de la RDC ». Criminologiquement parlant, la réflexion développée relève d'un mécanisme de « contrôle social » car, il s'agit, comme déjà affirmé, de l'esquisse d'un modèle de gestion du commandement là où celui-ci semble faire problème au regard - bien entendu des pesanteurs ci-dessus analysées.

Il convient d'abord de rappeler le fait que, relevant étymologiquement du domaine économique, le concept de management a acquis par la suite droit de cité dans d'autres domaines, notamment dans l'administration des affaires publiques et même dans les corps sécuritaires. Dans ces derniers, les pratiques de terrain montrent que le commandement usuel dans la plupart de ces corps relève principalement du " management dit par le haut ", c'est-à-dire une injonction à l'application stricte des principes règlementaires comme si l'agent d'exécution était, comme déjà affirmé, un automate dépourvu de toute marge d'appréciation et évoluant sur un terrain vierge, exempt de toute contrainte. Dans ce modèle, les dirigeants (ou commandants) recourent généralement à ce qu'ils ont appris dans des structures spécialisées de leur formation et rêvent de le voir (être) appliqué à la lettre.

A ce propos, nous pouvons encore rappeler la fameuse formule de commandement déjà évoquée d'un officier congolais gueulant sur son subalterne qui avait tendance à hésiter à l'ordre lui intimé :

«Oza raisonneur! Bomba mayele nayo na poche » [Tu raisonnes beaucoup! Cache ton intelligence dans la poche!]. Il s'agit là d'une belle illustration du commandement relevant du management par le haut.

Tout ceci pour dire que malgré ses connaissances, son expérience et sa maîtrise des éléments de terrain, le receveur de l'ordre n'a rien à dire, rien à suggérer au chef; son unique reflexe, c'est la simple exécution des ordres reçus. Et ceci corrobore encore mieux l'autre formule traditionnelle de commandement bien connue: " A l'ordre du chef, exécution d'abord et réclamation après! » [...] Lors de nos entretiens avec les agents commis à la sécurité, le premier syntagme de commandement qu'ils évoquaient, c'était celui-ci.

Le «management par le haut » présentant des limites manifestes relatives à la survalorisation de l'institutionnel et des ordres hiérarchiques intimés, on serait, vite tenté de lui substituer le modèle contraire, celui du « management par le bas » où c'est plutôt la réalité de terrain qui commanderait la conception et l'application des principes règlementaires. Ce qui est logiquement envisageable. Mais, une fois passé au peigne fin critique, ce deuxième modèle court facilement le risque de verser dans une exaltation démesurée des réalités pratiques de terrain au point d'être amené à croire que sans le terrain, rien ne serait envisa- 
geable, sans avoir scruté la réalité de terrain, aucun principe ne pourrait être initié, aune action ne pourrait être menée. Voilà qui crée un autre dilemme.

Fort de l'argumentaire avancée et des critiques développées, pour une gestion raisonnée du commandement dans les structures sécuritaires fonctionnant en RDC, nous suggérons un modèle de management que se distancie de deux précédents; il s'agit du modèle que nous qualifions - faute de meilleure expression - de « management pragma-institutionnel ${ }^{33}$ ». Pour Raoul KIENGE KIENGE ${ }^{34}$ (2005,50), le pragmatisme est à comprendre comme l'exigence de définir l'action à mener en tenant compte à la fois du contexte environnemental (ou local) et de plusieurs autres impératifs. En termes simples, il s'agit de l'adoption d'une attitude conséquente par rapport aux réalités de terrain, une attitude consciente des méfaits de l'automatisme. Par ailleurs, indique encore KIENGE KIENGE, le formel [1'institutionnel] signifierait que, malgré les contraintes humaines, structurelles et sociales, le sujet agissant ne procède pas à une totale table rase des tâches et/ou des rôles institutionnels lui assignés.

Par son principal trait caractéristique le pragma-institutionnel ou le pragma-formel se veut une forme de management où le commandement est conçu à l'intersection du modèle procédant «par le haut » et de celui procédant « par le bas ». L'intersection de ces deux modèles signifie que l'initiateur du commandement est d'abord un sujet professionnellement bien formé et lorsqu'il faut agir ou intervenir, il sait d'abord comment mettre en veilleuse $^{35}$ tout son a priori intellectuel, sentimental, professionnel et institutionnel afin de mieux saisir la situation en face telle qu'elle se présente. Et à l'intervention, il sait encore réactualiser ce qu'il a mis en veilleuse pour le croiser avec les pesanteurs de la réalité en face. Avec ce type de management, le commandement, vecteur principal de l'action sécuritaire, n'est plus pré-codé ou « robotisé », les politiques et les modes d'intervention sont « modelés » en fonction des situations concrètes à gérer. Il s'agit en quelque sorte d'un « management situationnel » qui concilie constamment, dans un élan on ne peut plus réaliste, les normativités institutionnelles et celles dites effectives [de terrain] avec toutes leurs contraintes et/ou contingences.

Bien qu'il y ait incontestablement référence à l'acquis appris en formation, ce modèle n'obéit pas à des schémas pratiques d'intervention préconçus. C'est en ceci qu'il se dit ou se veut pragmatique. Avec ce modèle, le commandement sécuritaire est une affaire des « têtes pensantes » aptes à la réflexion, à la créativité, à l'innovation et non l'apanage de n'importe quel Commandant-officier. Frédéric OCQUETEAU (2006:18) ${ }^{36}$ trouve que le

33 Cette expression est notre bricolage. On pourrait aussi dire « pragma-formel ». Pragma est le diminutif de pragmatique.

34 Raoul KIENGE KIENGE (2005), Le contrôle policier de la délinquance des jeunes à Kinshasa. Une approche ethnographique en criminologie, thèse de doctorat, Université Catholique de Louvain.

35 Il ne fait pas table rase.

36 Frédéric OCQUETEAU (2006), Mais qui donc dirige la police? Sociologies des Commissaires, Paris, Armand Colin. 
dirigeant [le commandant] doit réunir en une seule personne trois dimensions possibles du métier : celle de " flic de terrain », celle de " technicien expert en risque », ou celle d'un « manager de service » opérationnel ou administratif, et en même temps endosser la responsabilité d'une pluralité de rôles sociaux. Ce genre de dirigeant possède un leadership qui lui permet d'élaborer et de mettre en œuvre une vision organisationnelle pour son unité, au regard du mandat et des objectifs à atteindre. Celui-ci est conscient de la responsabilité qu'il $\mathrm{y}$ a à bien canaliser les énergies et les talents des hommes sous ses ordres. En outre, il doit être capable de représenter valablement son unité à l'extérieur tant devant les autorités hiérarchiques, administratives et les autres acteurs de l'espace social. Voilà qui exige encore des capacités de communication orale et écrite.

Avec le modèle du management pragma-formel, toute société imbue de progrès et de gestion saine des « désordres urbains » traiterait minutieusement toute la question relative à la nomination et à l'élévation des officiers sécuritaires aux différents postes de commandement. Les critères subjectifs tels que le clientélisme, les affinités ethniques, tribales, amicales, politiques et que sais-je encore, n'auraient aucune audience dans ces méandres.

Intervenant comme invité-conférencier dans notre cours de politique et pratiques de traitement de la délinquance, le commandant urbain [ville de Lubumbashi] de l'unité dite d'intervention rapide (PIR) ${ }^{37}$ nous a paru assez franc sur la délicatesse des interventions sécuritaires :

"Quand il y a désordre quelque part dans la ville, la hiérarchie nous confie des missions précises, des objectifs bien définis à atteindre et on nous demande d'agir. Comment faire? Là, on ne nous dit rien. Il peut s'agir, par exemple, de la libération d'un immeuble assiégé ou plutôt l'arrestation de certains malfrats. Il faut le faire... Avec les moyens mis à notre disposition, nous devons nous-mêmes «monter »les modes et les stratégies d'intervention. Mais bien sûr au départ, il faut maîtriser les techniques apprises. Moi, j'ai été formé comme commando d'intervention par les Français $[\ldots] »$.

Au chapitre des situations de " désordres » qu'il gère au quotidien, notre invité a évoqué notamment les violences au stade lors des grands matchs de football, la révolte des étudiants, les opérations nocturnes contre les bandits à main-armée, les marchés pirates, la traque des évadés de prison, les manifestations politiques, les mouvements des creuseurs miniers indépendants, etc. Il faut dire que ces creuseurs miniers, aux profils diversifiés [soldats démobilisés, évadés de prison, jeunes de la rue ou jeunes Kuluna, simples chômeurs...], représentent une redoutable force sociale, difficile à mâter quand elle se soulève. Interrogé par nous lors de son passage à l'école de criminologie à la recherche de ses relevés de côtes, un autre officier de la police de mines, notre ancien étudiant, s'est confié à nous en ces termes : 
"Là où nous sommes [sur le site minier], c'est comme au front pendant la guerre. Quand les pilleurs des minerais viennent dans la concession qui leur est interdite, ils sont bien préparés et bien armés. C'est la vie ou la mort. Ou bien, c'est toi qui meurs ou c'est eux. On n'est jamais à l'aise là, j'ai même perdu un peu de ma santé. Bon, c'est le boulot, on est là et ça marche [...]».

Lorsque cet officier nous confiait ces propos, il venait de se passer, quelques jours auparavant, des émeutes meurtrières dans ledit site suite à la délocalisation forcée des exploitants miniers artisanaux. Notre interviewé s'est abstenu de tout commentaire sur cet événement.

Pour corroborer les propos de nos enquêtés, LUNSUA KINZENZE (2009) ${ }^{38}$ trouve que la police, en tant qu'organisation est animée par des hommes, «des managers appelés commandants d'unité ». Lorsqu'il s'agit de rendre compte d'un événement impliquant la police, le langage médiatique utilise des expressions comme « la police est intervenue », " la police a ouvert une enquête ", " la police a tiré sur des manifestants », " la police a arrêté », etc. Les actions menées sont plus imputées à l'institution en tant que corps et non aux individus qui la composent. La loi elle-même, soutient encore LUNSUA, va dans le même sens en confiant des missions à la police et non au policier. Mais derrière ces actions relatées ou attendues d'un être indéterminé, il y a des individus, acteurs de chair et d'os qui interprètent leurs rôles, qui prennent des décisions, qui posent des actes et qui en endossent la responsabilité pénale ou disciplinaire à titre individuel. Ces acteurs sont ainsi appréciés en termes de performance et jouent chacun leur carrière individuelle. Alors au final, se demande le même chercheur, les moyens disponibles pour la police ne le sont-ils pas concrètement pour les commandants? Les contraintes imposées à l'institution policière ne sont-elles pas, en réalité, imposées à ses dirigeants? Plus précisément encore, les missions confiées à une unité ne sont-elles pas visiblement confiées au commandant?

Sans nul doute, la tâche du commandant est énorme. En pareille situation, qui recourrait aux formules pré-codées ou stéréotypées? Qui commanderait à la manière d'un robot? Voilà qui, à notre avis, appelle un commandement pragmatique-formel bien sûr au contenu et à la forme fluides, car appelant une perpétuelle contextualisation. Notre réflexion relève, en quelque sorte de l'intervention clinique en matière sécuritaire considérant que chaque situation en face, avec ses tenants et ses aboutissants, induirait des mesures appropriées. Il nous semble logique, pour ceux qui ont la charge de commander, de sortir du système figé du genre « copier-coller » ou de l'automatisme dans l'administration des ordres aux subalternes sous leur commandement. Il est probable que pareil modèle puisse trouver une assise fonctionnelle dans les sociétés où les initiatives privées sont véritablement encouragées et soutenues, car stimulant bien l'esprit d'inventivité et d'imagination.

On pourrait nous taxer de verser dans des « utopies »; s'il en serait le cas, il s'agirait alors des utopies qualifiées de « réalisables ». Lors du débat aux journées scientifiques susmentionnées, le commandant provincial de la police de circulation routière au Katanga 
[commissaire supérieur en grade] a déclaré séance tenante ${ }^{39}$ : "Professeur, mais ce que vous proposez là, moi je l'applique déjà avec mes collaborateurs! ». Voilà un feed back qui présage un début de compréhension et/ou d'appropriation du modèle encore en phase de conceptualisation et d'expérimentation. Heureusement que cette tâche ne reste pas l'apanage du seul initiateur du modèle.

En guise de synthèse, force sera donc de reconnaître que le pragma-formel prône un commandement inventif exigeant à la fois à l'entité dirigeante [qui commande] une compétence avérée et un savoir-faire bien ancré dans l'empirie. Si on nous concédait de rêver un peu, peut-être que la nouvelle vague d'officiers de police et militaires formés à l'Ecole de criminologie de l'Université de Lubumbashi, forts de leur compétence professionnelle et de l'expérience empirique, pourrait constituer le premier noyau « rare » de ces commandants au profil triple dont parle Frédéric OCQUETEAU ${ }^{40}$, commandants dont la RDC a besoin pour bien asseoir sa gouvernance sécuritaire. L'esquisse du modèle managérial que nous proposons se présente aussi comme une occasion privilégiée de matérialisation de l'« autonomie policière » chère à Dominique MONJARDET (1996), une autonomie qui ne se confond, certes pas, à l'arbitraire mais qui ouvre plutôt la voie à une inventivité pratique renvoyant à une autre notion de "capacité d'adaptation " chère à De VALKNEER et Vincent FRANCIS $(2007,15)^{41}$ pour qui « la police [en tant que corps sécuritaire] n'est pas seulement un bras armé du pouvoir; elle peut faire preuve d'adaptabilité là où la loi se révèle imperméable. Elle peut introduire du flou, de l'élasticité et même déplacer les frontières $»$.

Naturellement le modèle pragmatique-formel de gestion du commandement que nous suggérons n'est pas exempt de danger : dans une société fortement caractérisée par des pratiques et des normativités illicites ${ }^{42}$, l'inventivité managériale attendue des " commandants » court le risque de s'ériger en système " officiel » du seul « self- service ». L'intérêt de la structure professionnelle et celui de la nation peuvent se voir détournés ou sacrifiés au profit des intérêts individuels. Des garde-fous audit modèle sont donc à envisager, notamment par la véritable éducation à la citoyenneté qui doit, en principe, être bien inculquée, en priorité, dans les hautes sphères de commandement.

A ce stade de réflexion, nous voulons en toute franchise, reconnaitre que le modèle proposé n'a pas qualité d'outil managérial qui se prêterait à une application panacée; c'est juste une ébauche d'idées à consolider théoriquement. Si débat s'ensuit, notre objectif est atteint.

CONCLUSION

En fin de compte, nous concluons notre propos avec Jean-Noël TREMBLAY (2003) ${ }^{43}$ qui trouve finalement que la compétence des dirigeants doit se mesurer à la manière dont

39 Nous prenons l'organisation des dites journées [la direction de l'ECOCRIM/UNILU] à témoin.

40 « Flic de terrain », « technicien expert en risque » et « manager de service».

41 De VALKNEER et FRANCIS V. (2007), Manuel de sociologies policières, Bruxelles, Larcier.

42 Nous voulons dire les pratiques de corruption.

43 Jean-Noël TREMBLAY (1997), op.cit. 
ils adapteront la structure formelle de l'organisation du travail aux règles de structure paradoxalement informelles qui régissent le comportement des acteurs sur le terrain. Diriger n'est donc plus un acte de la raison abstraite, il n'est pas non plus un acte inconscient, mais plutôt une manifestation de la conscience. Voilà qui appelle des options managériales bien réfléchies.

\section{BIBLIOGRAPHIQUE}

1. De VALKNEER et FRANCIS V. (2007), Manuel de sociologies policières, Bruxelles, Larcier.

2. Dominique MONJARDET (1996), Ce que fait la police. Sociologie de la force publique, Paris, La Découverte.

3. Jean-Noël TREMBLAY (1997), Le métier de policier et le management, Canada, Presses de l'Université de Laval.

4. Jean-Paul BRODEUR, et Dominique MONJARDET (2003), « connaître la police » dans BRODEUR, J.P. et MONJARDET, D. (dir), Connaitre la police. Grands textes de la recherche anglo-saxonne, Paris, IHESI, pp. 9-21.

5. Luc ALBARELLO (2003), Apprendre à chercher. L'acteur social et la recherche scientifique, Bruxelles, De Boeck, $2^{\mathrm{e}}$ édition.

6. LUPITSHI WA NUMBI (2006), Les pratiques non règlementaires dans le réseau automobile des transports en commun de Lubumbashi, Mémoire de DEA en criminologie, Université de Lubumbashi.

7. LUNSUA KINZENZE (2009), La restructuration des relations dans une unité de police. Un mode de gestion du commandement, mémoire de licence en criminologie, Ecole de criminologie, Université de Lubumbashi (RDC).

8. Paolo NAPOLI (2003), Naissance de la police moderne, Paris, La Découverte.

9. Raoul KIENGE KIENGE (2005), Le contrôle policier de la délinquance des jeunes à Kinshasa. Une approche ethnographique en criminologie, thèse de doctorat, Université Catholique de Louvain.

10. Frédéric OCQUETEAU (2006), Mais qui donc dirige la police? Sociologies des Commissaires, Paris, Armand Colin.

11. Robert CASTEL (1990), «L'application de la loi : l'ordre des interactions et l'ordre des déterminations », in DIGNEFFE, F. (dir), Acteur social et délinquance. Une grille de lecture du système de justice pénale, Bruxelles/Liège, Mardaga, Coll. Psychologie et Sciences humaines, pp.295 - 303.

12. Tréfon THEODORE (2007), Parcours administratifs dans un Etat en faillite. Récits populaires de Lubumbashi (RDC), Paris, L'Harmattan.

13. TSHINYAMA, KADIMA (2009), L'observation ethnographique d'un commissariat à Lubumbashi. Une compréhension des pratiques policières en contexte congolais, Thèse de doctorat en criminologie, Université de Lubumbashi (RDC).

14. TSHINYAMA KADIMA (2011), « comprendre les pratiques policières en contexte congolais. Une étude ethnographique réalisée dans un commissariat de Lubumbashi ", in Déviance et société, La charte. 